\title{
THE TRANSMISSION DYNAMICS OF CANINE AMERICAN CUTANEOUS LEISHMANIASIS IN HUÁNUCO, PERU
}

\author{
RICHARD REITHINGER, JUAN CANALES ESPINOZA, AND CLIVE R. DAVIES \\ Disease Control and Vector Biology Unit, Department of Infectious and Tropical Diseases, London School of Hygiene and Tropical \\ Medicine, London, United Kingdom; Dirección Regional de Salud Huánuco, Huánuco, Peru
}

\begin{abstract}
The epidemiology of canine American cutaneous leishmaniasis (ACL) due to Leishmania (Viannia) spp. was investigated in Huánuco, Peru to 1) describe the natural course of canine L. (Viannia) infections and 2) assess the role of domestic dogs as ACL reservoir hosts. Over a three-year period 1,022 dogs were surveyed, with cumulative village L. (Viannia) prevalence being $26 \%$ (range $=0-100 \%$ ). The incidence of $L$. (Viannia) was estimated to be 0.285 dogs/year (95\% confidence interval $[\mathrm{CI}]=0.160-0.410)$ using cross-sectional data and 0.291 dogs/year $(95 \% \mathrm{CI}=$ 0.195-0.387) using data from 108 dogs that were surveyed prospectively. The recovery rate was estimated to be 0.456 dogs/year $(95 \%$ CI $=0.050-0.862)$ and 0.520 dogs/year $(95 \% \mathrm{CI}=0.302-0.738)$, respectively. Using those findings, the basic reproduction number was estimated to be $\mathrm{R}_{0} \approx 1.9$; if dogs were the principal ACL reservoirs, the mean yearly effort (i.e., coverage or elimination) of a dog control intervention (e.g., collaring, culling, or vaccination) to ensure the elimination of L. (Viannia) spp. transmission would be as low as $47 \%$.
\end{abstract}

\section{INTRODUCTION}

The leishmaniases are a group of diseases transmitted to humans and animal hosts by the bite of phlebotomine sand flies. Leishmania (Viannia) braziliensis and L. (V.) peruviana cause American cutaneous leishmaniasis (ACL); whereas $L$. (V.) braziliensis is the most widespread of the L. (Viannia) spp. and distributed throughout Latin America, L. (V.) peruviana appears to be restricted to the inter-Andean valleys of Peru. ${ }^{1}$ American cutaneous leishmaniasis is characterized by single or multiple cutaneous lesions that have a variable tendency to self-cure. Although the parasites generally remain localized at the site of infection, they occasionally disseminate to cause disfiguring and potentially life-threatening mucocutaneous leishmaniasis.

Leishmania (Leishmania) infantum causes visceral leishmaniasis (VL) and is, so far, the only Leishmania sp. found in both New and Old World. Except in a few anthroponotic transmission foci, domestic dogs are the main reservoir hosts of VL. ${ }^{2}$ Because several studies reported high canine $L$. ( $\mathrm{Vi}$ annia) spp. infection rates, there is a growing belief that dogs are also the reservoirs of ACL as well; however, so far, evidence to support that claim has been circumstantial. ${ }^{3}$

Despite the extensive literature reporting canine L. (Viannia) infection, data on the clinical, parasitologic, and immunologic course of L. (Viannia) infection in dogs are scarce, and are restricted to four experimental studies, from which it is difficult to draw any conclusions due to differences in experimental protocol and small sample size. ${ }^{3}$ The course of infection following experimental parasite inoculation is also likely to differ from the course of infection due to natural infection via sand fly bite. The only information on the natural (sero) conversion and recovery rates of dogs infected with L. (Viannia) parasites comes from two field studies. In a small study in Brazil, 3 of 14 L. (V.) braziliensis-infected dogs were shown to heal spontaneously 2-3 months after parasite demonstration, with complete clinical and serorecovery; lesions and positive immunofluorescence antibody test results reappeared in all three dogs during the 11-month study followup. ${ }^{4}$ Recently, a prospective survey in a $L$. (V.) braziliensisendemic area in Argentina reported yearly incidence rates of $12 \%$ (6 of 52) and 19\% (10 of 52) for clinical disease (i.e., appearance of ACL lesions) and infection (i.e., as diagnosed by an enzyme-linked immunosorbent antibody test [ELISA]), respectively; no information on serorecovery rates was reported. $^{5}$

Comparatively vast is the literature describing the transmission dynamics of canine VL, with studies showing that 1) dogs are highly susceptible to $L$. infantum with high infection rates observed throughout the $L$. infantum-endemic range;, 2) susceptibility to and persistence of $L$. infantum infection is dependent on host genetic background (e.g., pure breed dogs are more susceptible than mongrel $\operatorname{dogs})^{7}$ and dog ecology (e.g., rural or hunting dogs have a higher risk of VL infection than urban or pet dogs, respectively);,8 and 3) once infected, dogs tend to remain parasitologically positive for life and thus, infectious to the sand fly vector. ${ }^{9}$

The objectives of the study reported here were to 1) describe the natural course of L. (Viannia) infection in dogs parasitologically and immunologically by estimating incidence and recovery rates using data from both cross-sectional and prospective surveys; and 2) assess the epidemiologic role of dogs in the L. (Viannia) transmission cycle in Huánuco, Peru.

\section{MATERIALS AND METHODS}

Study site. All surveyed dogs came from 18 villages in the Department of Huánuco $\left(10^{\circ} 50^{\prime} \mathrm{S}, 76^{\circ} 10^{\prime} \mathrm{W}\right)$, an $\mathrm{L}$. (V.) braziliensis and $L$. (V.) peruviana-endemic area in Peru where the London School of Hygiene and Tropical Medicine and the Universidad Peruana Cayetano Heredia have been carrying out an epidemiologic study on human ACL (Davies CR, Llanos-Cuentas A, unpublished data): ${ }^{10-12}$ Pomacucho (Code 901), Conchumayo (902), Quechualoma (903), Chinobamba (904), Vilcabamba (905), Limapampa (906), Chullay (907), Coz (908), Huancapallac (909), Virroy (914), Cochachinche (915), Parcoy (916), Atahuayón (917), Mauca (918), Cochatamba (919), El Rancho (920), La Esperanza (921), and San Rafael (922). For comparative analyses, villages were grouped into five different valleys (V): V1 $(901,902)$, V2 (903, 904, 905), V3 (906, 909), V4 (907, 908) and V5 (914-919). Villages 920-922 were not included in the analyses because they were isolated villages, with a too low number of dogs sampled for analytical purposes (Figure 1). There are no re- 


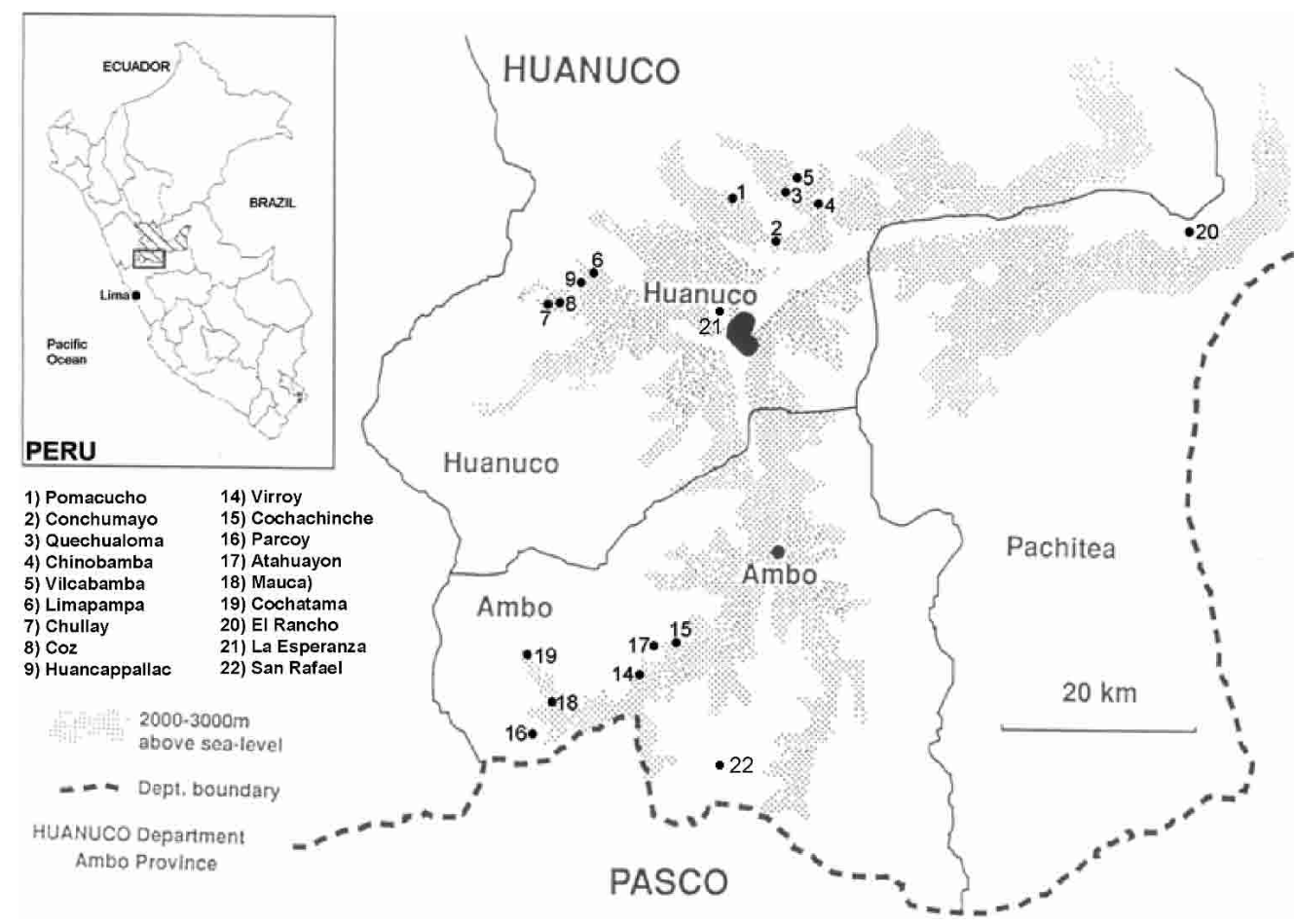

Figure 1. Map of the Leishmania (Viannia)-endemic study site in and near Huánuco, Peru. The town and villages are indicated. $\mathrm{m}=\mathrm{meters}$.

ported L. (L.) infantum or Trypanosoma cruzi infections in the study area.

Dog sampling and diagnosis. Dogs were surveyed either by house-to-house visits or gathered in the village square during rabies vaccination campaigns carried out by the local Ministry of Health. None of the dogs belonged to a recognizable breed and all were guard or hunting dogs; dog age was obtained by interview of owners or by observing tooth decay. In follow-up visits, owners were asked whether previously surveyed dogs were still alive or had died. Dogs were surveyed at approximately yearly intervals between 1997 and 1999, and ACL was diagnosed clinically, parasitologically (i.e., by a polymerase chain reaction [PCR]) or immunologically (i.e., by an ELISA) as previously described, with dogs diagnosed as ACL positive when positive by either PCR, ELISA, or both (i.e., infection). ${ }^{10,11}$ The sensitivity of the diagnostic tests used was $31 \%$ and $81 \%$ for the PCR and ELISA, respectively; specificity was $100 \%$ for both tests. ${ }^{11}$ Due to logistical constraints, V3 and V5 were only sampled during one and two years, respectively.

Data analysis: cross-sectional surveys. The instantaneous per capita conversion (i.e., force of infection $[\lambda]$ ) and recovery $[\rho]$ rates were estimated from age-prevalence data where the proportion of animals positive at age $a, P(a)$, is given by

$$
P(a)=\frac{\lambda}{(\lambda+\rho)} \cdot\left(1-e^{-(\lambda+\rho) a}\right)
$$

This equation assumes that 1) $\lambda$ and $\rho$ are age independent and constant over time, 2) individuals in the host population are homogeneously exposed, 3) individuals who are infected become instantaneously positive, and 4) the association between age and prevalence is observed at equilibrium. $\lambda$ and $\rho$ can then be estimated by maximum likelihood using observed age-prevalence data. ${ }^{13}$

Data analysis: prospective surveys. The instantaneous incidence rate among the susceptible dog population was estimated from the frequency of conversions from a negative to a positive PCR or ELISA (i.e., $\mathrm{PCR}^{-}$and $\mathrm{PCR}^{+}$or $\mathrm{ELISA}^{-}$ and $\mathrm{ELISA}^{+}$, respectively) diagnosis during the intervals between the 1997, 1998, and 1999 surveys. Both $\lambda$ and $\rho$ can be derived from the prospective survey data by solving the simultaneous equations

$$
\begin{aligned}
& P(c)=\frac{\lambda}{(\lambda+\rho)} \cdot\left(1-e^{-(\lambda+\rho) t}\right) \\
& P(r)=\frac{\rho}{(\lambda+\rho)} \cdot\left(1-e^{-(\lambda+\rho) t}\right)
\end{aligned}
$$

Where $P(c)$ is the proportion of individuals converting from a negative to a positive diagnosis in time $t$, and $P(r)$ is the proportion of individuals converting from a positive to a negative diagnosis.

\section{RESULTS}

Dog demography. During the three-year study period 1,114 dogs were surveyed, of which 1,022 $(1997=481,1998=284$, $1999=257)$ were sampled and diagnosed by both the ELISA and PCR; ${ }^{10,11} 138$ (14\%) of 1,022 samples came from 108 dogs that were sampled more than once. Of the 1,022 dogs included in the analyses, 418 (41\%) and 582 (57\%) were female and male, respectively; 22 dogs were not sexed (Tables 1 and 2). Mean dog age for the whole study period was 30 months (range $=1-180)$. The life expectancy $(L)$ of dogs was calcu- 


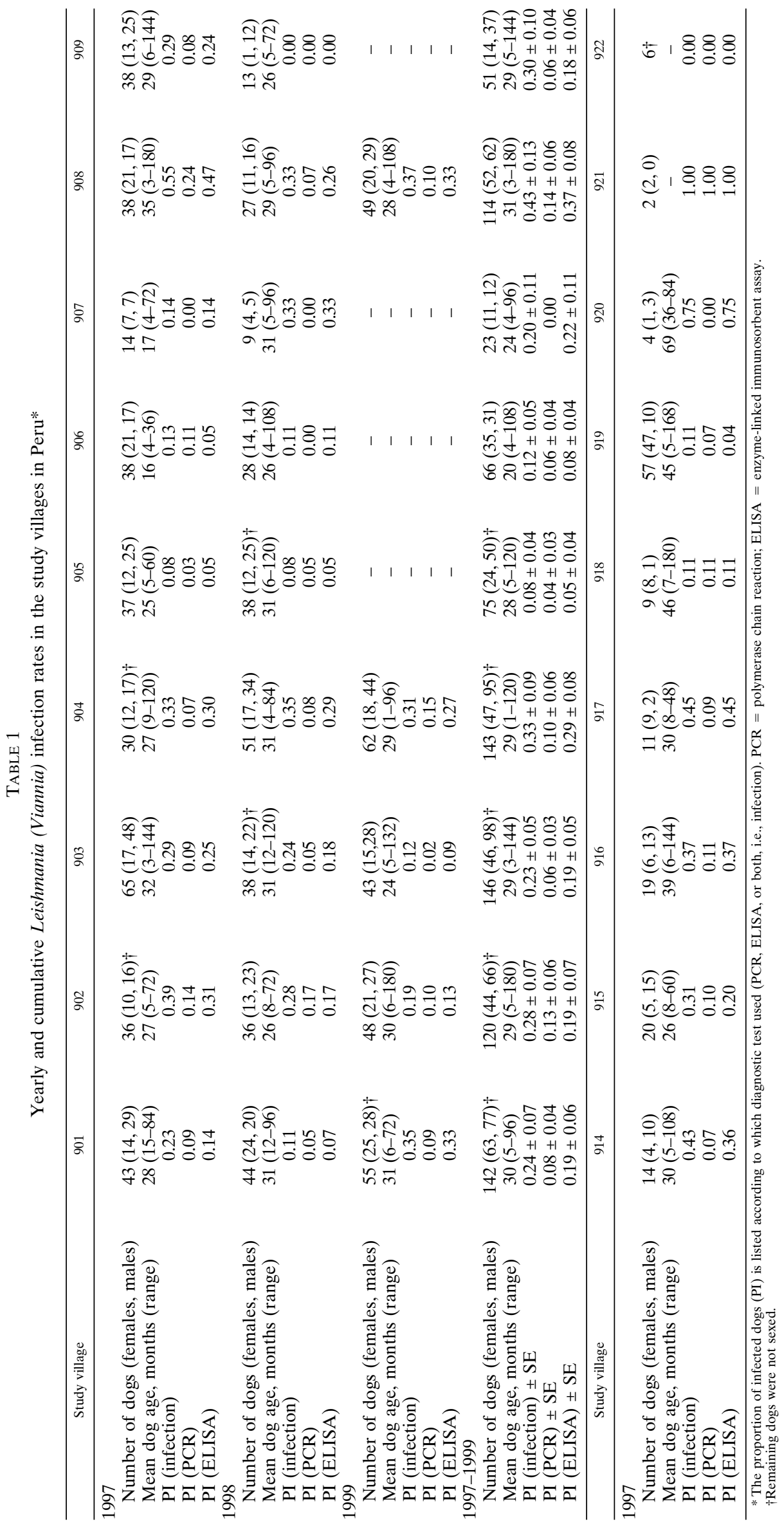


TABLE 2

Yearly and cumulative Leishmania (Viannia) infection rates in the study valleys in Peru*

\begin{tabular}{|c|c|c|c|c|c|c|}
\hline Study valley & V1 & $\mathrm{V} 2$ & V3 & $\mathrm{V} 4$ & V5 & All villages \\
\hline \multicolumn{7}{|l|}{1997} \\
\hline PI (infection) $\dagger$ & 0.30 & 0.24 & 0.21 & 0.44 & 0.24 & 0.27 \\
\hline PI (PCR) & 0.11 & 0.07 & 0.09 & 0.17 & 0.08 & 0.10 \\
\hline PI (ELISA) & 0.22 & 0.20 & 0.14 & 0.38 & 0.28 & 0.22 \\
\hline \multicolumn{7}{|l|}{1998} \\
\hline PI (infection) $\dagger$ & 0.19 & 0.24 & 0.07 & 0.36 & - & 0.21 \\
\hline PI (PCR) & 0.10 & 0.06 & 0.00 & 0.06 & - & 0.06 \\
\hline PI (ELISA) & 0.11 & 0.19 & 0.07 & 0.31 & _- & 0.17 \\
\hline \multicolumn{7}{|l|}{1999} \\
\hline PI (infection) $\dagger$ & 0.27 & 0.23 & - & 0.37 & - & 0.27 \\
\hline PI (PCR) & 0.10 & 0.10 & - & 0.10 & - & 0.10 \\
\hline PI (ELISA) & 0.23 & 0.19 & _- & 0.33 & _- & 0.24 \\
\hline \multicolumn{7}{|l|}{$1997-1999$} \\
\hline Number of dogs (females, males) & $262(107,143) \ddagger$ & $364(117,243) \ddagger$ & $117(49,68)$ & $137(63,74)$ & $130(79,51)$ & $1022(418,582) \ddagger$ \\
\hline Mean dog age, months (range) & $29(5-180)$ & $29(1-144)$ & $24(4-144)$ & $29(3-180)$ & $38(5-180)$ & $30(1-180)$ \\
\hline $\mathrm{PI}$ (infection) $\dagger \pm \mathrm{SE}$ & $0.26 \pm 0.05$ & $0.24 \pm 0.04$ & $0.16 \pm 0.04$ & $0.39 \pm 0.07$ & $0.24 \pm 0.04$ & $0.26 \pm 0.02$ \\
\hline $\mathrm{PI}(\mathrm{PCR}) \pm \mathrm{SE}$ & $0.10 \pm 0.03$ & $0.07 \pm 0.02$ & $0.06 \pm 0.03$ & $0.12 \pm 0.04$ & $0.08 \pm 0.02$ & $0.09 \pm 0.01$ \\
\hline $\mathrm{PI}($ ELISA) $\pm \mathrm{SE}$ & $0.19 \pm 0.04$ & $0.19 \pm 0.03$ & $0.12 \pm 0.04$ & $0.34 \pm 0.07$ & $0.18 \pm 0.03$ & $0.20 \pm 0.02$ \\
\hline$\lambda \pm \mathrm{SD}$ & $0.35 \pm 0.12$ & $0.19 \pm 0.04$ & $0.12 \pm 0.04$ & $0.84 \pm 0.34$ & $-\S$ & $0.29 \pm 0.04$ \\
\hline$\rho \pm \mathrm{SD}$ & $0.76 \pm 0.22$ & $0.17 \pm 0.05$ & $-\S$ & $0.97 \pm 0.44$ & $-\S$ & $0.46 \pm 0.06$ \\
\hline L (vears) & 5.36 & 3.51 & 1.81 & 1.81 & $-\S$ & 3.04 \\
\hline $\mathrm{R}_{0}$ & 2.88 & 1.67 & 1.22 & 2.52 & $-\S$ & 1.88 \\
\hline
\end{tabular}

lated from the cohort of dogs followed-up between 1997 and 1999 according to the following equations

$$
\begin{gathered}
\delta=-\ln \left(1-\frac{n_{t+m}}{N_{t}}\right) \cdot \frac{12}{\left(m / n_{t+m}\right)} \\
L=\frac{1}{\delta}
\end{gathered}
$$

where $\delta$ is the mortality rate per year, $n_{t+m}$ is the number of dogs that died $m$ months after time $t$ (i.e., the first time dogs entered the cohort), $N_{t}$ is the total number of dogs at time $t$, and $m$ the total time in dog-months of cohort dogs that died between times $t$ and $t+m$. Follow-up data was available for 333 dogs, 122 of which died between any two surveys after a total 2,032 dog-months. The total mortality rate was 0.329 dogs/year (95\% confidence interval $[\mathrm{CI}]=0.227-0.452)$; thus, the life expectancy was 3.04 years $(95 \% \mathrm{CI}=2.21-3.61$, range between study valleys $=1.81-5.36)$. A higher mortality rate was observed in ACL-infected dogs (0.399 dogs/year, $95 \%$ CI $=0.301-0.497, \mathrm{n}=47)$ than in non-infected dogs (0.298 dogs/year, 95\% CI $=0.237-0.357, \mathrm{n}=75)$; thus, the life expectancy of an ACL-infected dog was 2.51 years $(95 \%$ $\mathrm{CI}=2.01-3.32)$ compared with 3.36 years $(95 \% \mathrm{CI}=$ 2.80-4.22) for a non-infected dog (Yates-corrected $\chi^{2}=2.35$, $P=0.13)$.

Cross-sectional data. We have previously demonstrated that L. (Viannia) infection was detected in 26\% (262 of 1,022) of sampled dogs either by the PCR or ELISA; 9\% (90 of $1,022)$ and $20 \%$ (209 of 1,022) were positive by the PCR or ELISA, respectively (Tables 1 and 2). ${ }^{11}$ The proportion of infected dogs was significantly different between valleys for the total three-year study period (Yates-corrected $\chi^{2}=20.13$, degrees of freedom $[\mathrm{df}]=17, P<0.001)$, in $1997\left(\chi^{2}=10.85\right.$, $\mathrm{df}=4, P=0.028)$ and $1998\left(\chi^{2}=10.14\right.$, df $\left.=3, P=0.017\right)$, but not in $1999\left(\chi^{2}=3.25, \mathrm{df}=2, P=0.2\right)$. Although a lower proportion of infected dogs was observed in all study villages in 1998 compared with 1997 or 1999, this difference was not significant $\left(\chi^{2}=3.57, \mathrm{df}=2, P=0.17\right)$. In any one sampling year, prevalence of infection was highest in V4 and lowest in $\mathrm{V} 3$, with observed cumulative prevalence of infection being as high as $0.44 \pm 0.07$ (proportion infected \pm SE) (1997) and 0.07 \pm 0.03 (1998), respectively. The highest observed canine ACL prevalence observed during the three years of the study was in village 908 in 1997, where 21 of $38(0.55 \pm 0.08)$ dogs were ACL positive. No ACL-infected dogs in surveyed animals were found on two occasions only, in villages 909 (in 1998) and 922 (in 1997) (Table 1).

Using maximum likelihood, we estimated the instantaneous incidence $(\lambda \pm \mathrm{SE})$ and recovery $(\rho \pm \mathrm{SE})$ rates from the cumulative ACL prevalence data, according to the diagnostic criteria used, i.e., dogs diagnosed as $\mathrm{PCR}^{+}, \mathrm{ELISA}^{+}$, or both (Table 1). The estimated instantaneous incidence rate for infection (i.e., dogs either ELISA ${ }^{+}, \mathrm{PCR}^{+}$, or both) $\lambda_{\text {infection }}$ was $0.285 \pm 0.064 \mathrm{dogs} / \mathrm{year}$ and the recovery rate for infection $\rho_{\text {infection }}$ was $0.456 \pm 0.207$ dogs/year (Table 3 and Figure 2). Estimates of $\lambda_{\text {infection }}$ and $\rho_{\text {infection }}$ varied between valleys and were as high as $0.83 \pm 0.34$ dogs/year and $0.97 \pm 0.44 \mathrm{dogs} /$ year, respectively, in V4 (Table 2).

Prospective data. A total of 138 observations were used in the analyses: 49 dogs were surveyed in 1997 and 1998, 33 dogs in 1998 and 1999, 16 dogs in 1997 and 1999. Twenty dogs were sampled at all three time points. Among the 138 observations, $11(9.0 \%)$ of 122 dogs converted from a $\mathrm{PCR}^{-}$to a $\mathrm{PCR}^{+}$ result during two surveys, and $8(50 \%)$ of 16 recovered from a $\mathrm{PCR}^{+}$to a $\mathrm{PCR}^{-}$result. Twenty $(17.7 \%)$ of 113 dogs seroconverted during any two surveys and 11 (44.0\%) of 25 serorecovered. Using diagnostic criteria set out previously, ${ }^{11}$ we determined that $23(22.3 \%)$ of 103 dogs were incident Leish- 
TABLE 3

Epidemiology of Leishmania (Viannia) in dogs: incidence and recovery rates estimated from cross-sectional and prospective surveys*

\begin{tabular}{|c|c|c|c|}
\hline & Infection $\dagger$ & PCR & ELISA \\
\hline \multicolumn{4}{|l|}{ Incidence $(\lambda \pm \mathrm{SE})$} \\
\hline Cross-sectional data & $0.285 \pm 0.064$ & $0.147 \pm 0.008$ & $0.180 \pm 0.041$ \\
\hline Prospective data & $0.291 \pm 0.049$ & $0.113 \pm 0.028$ & $0.227 \pm 0.041$ \\
\hline \multicolumn{4}{|l|}{ Recovery $(\rho \pm \mathrm{SE})$} \\
\hline Cross-sectional data & $0.456 \pm 0.207$ & $1.237 \pm 1.018$ & $0.288 \pm 0.176$ \\
\hline Prospective data & $0.520 \pm 0.111$ & $0.630 \pm 0.181$ & $0.565 \pm 0.137$ \\
\hline
\end{tabular}

mania infections during any two surveys, while 14 (40.0\%) of 35 dogs had infections that resolved. Thus, solving equations 2 and 3 (using the average time between survey time points), the instantaneous rates of conversion and recovery for infection were $\lambda_{\text {infection }}=0.291 \mathrm{dogs} /$ year $(95 \% \mathrm{CI}=$ $0.195-0.387)$ and $\rho_{\text {infection }}=0.520 \mathrm{dogs} /$ year $(95 \% \mathrm{CI}=$ $0.302-0.738$ ), respectively (Table 3 ).

To better understand the dynamics of L. (Viannia) transmission in dogs, incidence and recovery rates were estimated in a four-compartmental model for different status of disease with 12 possible rates defining the movements between pairs of compartments. At any time point, each dog in the cohort can be in one of four states because they may have been either $\mathrm{PCR}^{-}$or $\mathrm{PCR}^{+}$and either $\mathrm{ELISA}^{-}$or $\mathrm{ELISA}^{+}$(Figure 2). As before, the parameters describing the yearly rates were derived using equations 2 and 3. Whereas no difference was observed between the seroconversion rates of $\mathrm{PCR}^{-}$dogs (i.e., 0.192 dogs/year) and in $\mathrm{PCR}^{+}$dogs (i.e., $0.228 \mathrm{dogs} /$ year), the parasitologic conversion rates of ELISA $^{-}$dogs (i.e., $0.072 \mathrm{dogs} /$ year) was half the rate in $\mathrm{ELISA}^{+}$dogs (i.e., 0.156 dogs/year) (Figure 2). The serologic recovery rate was higher in $\mathrm{PCR}^{-}$dogs (i.e., 0.642 dogs/year) than in $\mathrm{PCR}^{+}$dogs (i.e., $0.384 \mathrm{dogs} /$ year). Similarly, the parasitologic recovery rate was much higher in ELISA ${ }^{-}$dogs (i.e., 1.032 dogs/year) than ELISA $^{+}$dogs (i.e., 0.168 dogs/year). The odds of susceptible (i.e., $\mathrm{PCR}^{-}$ELISA $^{-}$) dogs becoming serologically positive was significantly higher than the odds of dogs becoming parasitologically positive (odds ratio $[\mathrm{OR}]=3.34,95 \% \mathrm{CI}$

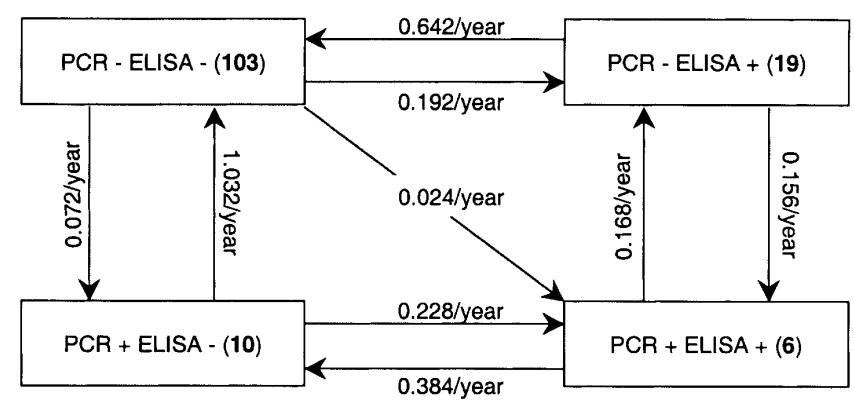

Figure 2. Compartmental model of Leishmania (Viannia) transmission in dogs. Incidence rates $(\lambda)$ and recovery rates $(\rho)$ were calculated using prospective data and solving equations E2 and E3 (see Materials and Methods). At any one time, there are four possible states cohort dog could have been in: $\mathrm{PCR}^{-}$or $\mathrm{PCR}^{+}$and $\mathrm{ELISA}^{-}$or ELISA $^{+}$. Numbers in parentheses represent the number of dogs in each state. $\mathrm{PCR}=$ polymerase chain reaction; ELISA = enzymelinked immunosorbent assay.
$=1.08-11.02$, Yates-corrected $\left.\chi^{2}=4.49, P=0.034\right)$, or becoming both serologically and parasitologically positive (OR $=5.68,95 \% \mathrm{CI}=1.48-25.60$, Yates-corrected $\chi^{2}=7.37$, $P=0.007)$.

When we used difference equations derived from the incidence and recovery rates in the compartmental model, the change in the proportion of dogs in each of the four states can be simulated over very short time intervals; as at time 0 , all dogs will be susceptible to infection ( $\mathrm{PCR}^{-} \mathrm{ELISA}^{-}$) (Figure $3 \mathrm{E})$. The simulations show that dog-age prevalence and simulations from the prospective data are remarkably consistent with the rates that were calculated from the cross-sectional data using maximum likelihood (Figure 3A-C).

Basic reproduction number $\left(\mathbf{R}_{\mathbf{0}}\right)$. The basic reproduction number $R_{o}$ can be estimated as

$$
R_{0}=1+\frac{L}{A}
$$

where $L$ is the life expectancy of a $\operatorname{dog}$ (3.04 years, 95\% CI = 2.21-3.61) and $A$ is the average time for a dog to acquire infection which can be estimated from the reciprocal of the force of infection (i.e., $\left.1 / \lambda_{\text {infection }}\right) \cdot{ }^{14}$ Using cross-sectional and prospective data (Table 3), we estimated that $A=3.51(95 \%$ $\mathrm{CI}=2.44-6.25)$ and 3.44 years $(95 \% \mathrm{CI}=2.58-5.13)$, respectively. Thus, $R_{o}$ is estimated to be 1.87 and 1.88 , respectively.

\section{DISCUSSION}

This is the first large-scale study to describe the epidemiology of ACL in dogs. Using both cross-sectional and prospective data, we estimated the ACL incidence and recovery rates in the dog population surveyed in Huánuco. Although there was a slight difference in age-prevalence curves observed between years (Figure 3D), both cross-sectional and prospective analyses gave similar results with incidence and recovery rates were estimated to be $\approx 0.3 \mathrm{dogs} /$ year and $\approx 0.5$ dogs/year, respectively (Figures $3 \mathrm{~A}$ and $\mathrm{E}$ ). The reason why estimates from cross-sectional and prospective data were slightly different is because ACL transmission is likely to be heterogeneous, ${ }^{9,12,15}$ as shown by the different prevalence of infection and incidence rates between study villages, valleys and years (Tables 1 and 2); ${ }^{12}$ ACL transmission in Huánuco is seasonal, with a peak transmission season between May and August after the yearly rainy season (January to April). Other factors that will affect $L$. (Viannia) transmission rates between villages or valleys are temperature and altitude, factors that will influence sand fly vector ecology and thus, vectorial capacity. ${ }^{16}$

One major caveat of this study is that currently no diagnostic test exists that is able to differentiate between $L$. (V.) peruviana and L. (V.) braziliensis infections, both of which are endemic in Huánuco. Of note is the high recovery rates (as estimated by both cross-sectional and prospective data) observed in this study. A possible explanation for this is that dogs readily develop a cell-mediated Th1 immune response against L. (Viannia) parasites that controls infection (i.e., parasite numbers in the blood decrease below the level detectable by the PCR), since $\mathrm{PCR}^{+} \mathrm{ELISA}^{+}$dogs serorecover (i.e., they become ELISA $^{-}$) at a higher rate than they cure 
A

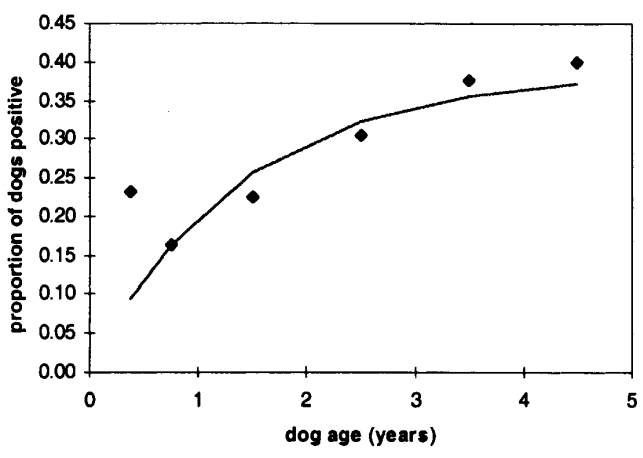

C

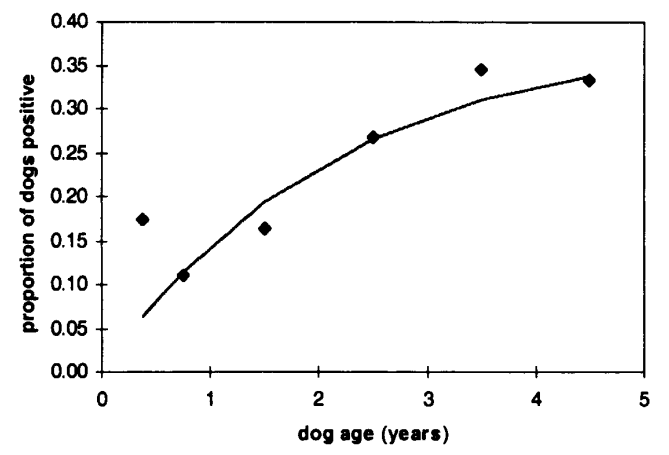

$\mathbf{E}$

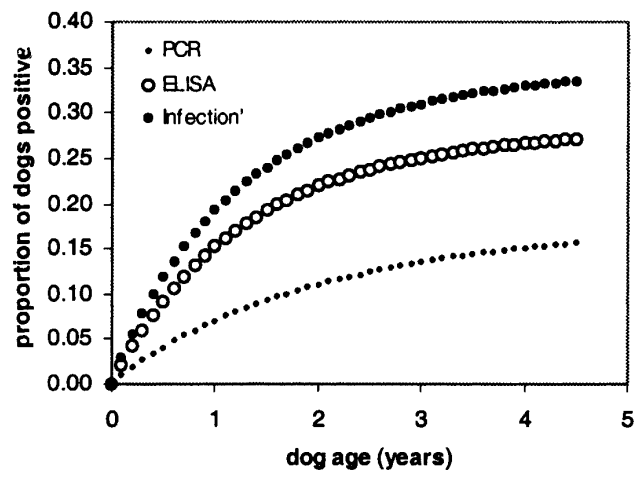

B

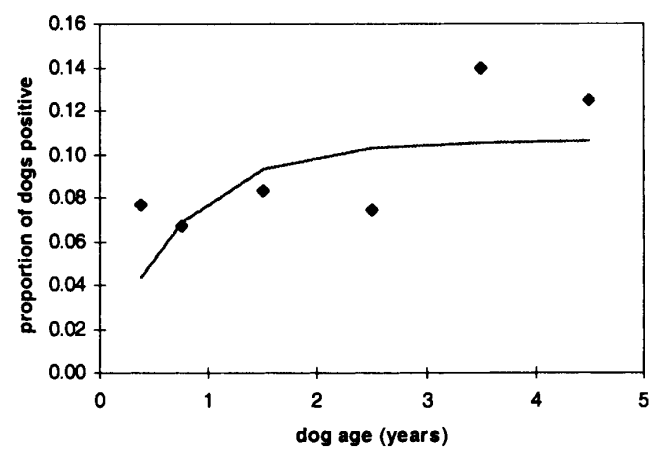

D

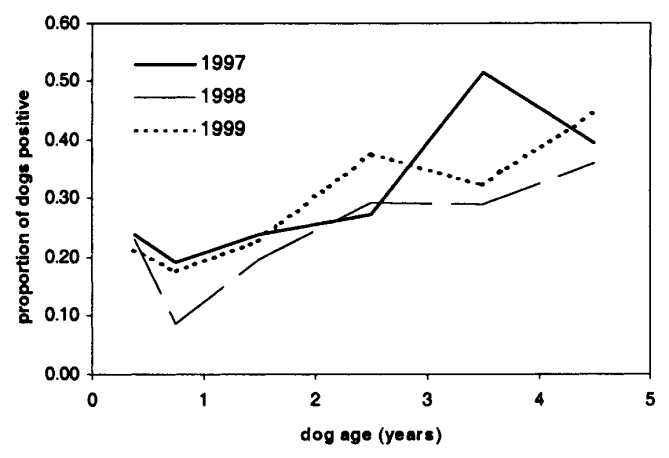

FIGURE 3. Cumulative age-prevalence of American cutaneous leishmaniasis infection in dogs according to diagnostic technique: infection, i.e., positive by enzyme-linked immunosorbent assay [ELISA] or polymerase chain reaction [PCR], or both) (A), PCR (B), ELISA (C). Lines are the best-fit to the data as estimated by maximum likelihood; for estimates of $\lambda$ and $\rho$ (see Table 3). Age-prevalence curves (infection) in different survey years (D). Using the incidence and recovery rates calculated from the prospective surveys, the cumulative age-prevalence was simulated (E).

parasitologically (i.e., becoming $\mathrm{PCR}^{-}$) (Figure 2). The high rates of serorecovery and parasitologic recovery could also be explained if a proportion of infections are due to $L$. (V.) peruviana. Leishmania (V.) peruviana is known to be very pathogenic, i.e., the parasites easily infect dogs, but unlike $L$. $(V$.$) braziliensis-infections, they self-cure and do not cause$ clinical disease. ${ }^{1,16}$ Both cross-sectional and prospective surveys indicate that $L$. (Viannia) parasites infecting dogs in Huánuco are pathogenic, but not very virulent, indicating that many infections may have been caused by $L$. (V.) peruviana. Cross-sectional surveys showed that $3.8 \%$ of the dogs had clinical disease and only one of the dogs with an incident infections (i.e., $4.3 \%$ ) was clinically positive, which is a relatively low proportion when compared with other studies re- porting ACL in dogs. ${ }^{3}$ No isolation of parasites was attempted in this study, but a previous study had shown that most (11 of 12) of the Leishmania dog isolates in the study villages are L. (V.) braziliensis/L. (V.) peruviana hybrids (Nolder D, 1998. Molecular Diversity in the Leishmania Subgenus Viannia. PhD Thesis. London, United Kingdom: University of London). It has to be noted that the course of $L$. (V.) braziliensis/L. (V.) peruviana hybrids in either natural hosts or experimental laboratory animals is unknown. Interestingly, the highest observed serorecovery rate (1.032 dogs/ year) was in those dogs that appeared to have low levels of parasites in their blood (i.e., $\mathrm{PCR}^{-}$ELISA $^{+}$dogs). This again indicates that dogs are able to clear Leishmania infections readily (as otherwise a sustained antibody response would be 
observed), underlining the avirulence of Leishmania parasites infecting dogs in Huánuco.

The observed high recovery rates are in stark contrast to recovery rates $(\approx 0.100$ person/year) observed in humans living in $L$. $(V$.$) peruviana-endemic areas, { }^{16}$ where infections tend to result in life-long immunity to the parasite. This lifelong immunity is probably sustained by continuous antigen presentation due to persistent parasites. ${ }^{17}$ The observed recovery rates are also in contrast with those observed in $L$. infantum-endemic areas, where most infections in dogs appear to be life-long with no detectable recovery. ${ }^{6,8,14}$ Nevertheless, the recovery rates that were detected in L. (Viannia)infected dogs mean that infection persists (i.e., $1 / \rho=2$ years) for almost the average lifetime of an ACL-infected dog (2.5 years). Thus, should a dog become infected at an early age, it will remain infected until death, and thus, potentially infectious to the sand fly vector, a key criteria to incriminate dogs as reservoirs of ACL. ${ }^{3}$

Canine ACL transmission dynamics are of interest because it allows the quantification (in terms of $\mathrm{R}_{0}$ ) of the putative reservoir role of domestic dogs. In epidemiologic terms, a primary reservoir of a zoonosis can be defined as a host that is able to maintain the endemic transmission of the pathogen in the absence of any other host species, i.e., maintain $R_{0}$ greater than $1 .^{3}$ Secondary reservoirs are those host species whose presence significantly increases $R_{0}$, but who are unable to maintain $R_{o}$ greater than 1 in the absence of other host species. In contrast, accidental reservoir hosts play no role in the transmission cycle and their presence has no impact on $\mathrm{R}_{0}$. Thus, were dogs primary ACL reservoir hosts, it would be feasible to eradicate domestic transmission by targeting dogs; but if dogs are secondary reservoir hosts, targeting dogs would only reduce (i.e., control) ACL. Of course, any dog control strategy would be ineffective were dogs accidental hosts of ACL.

If infected dogs are significantly infectious to sand flies and do play a role in the maintenance of $L$. (Viannia) transmission in Huánuco, they contribute an $\mathrm{R}_{0}$ of approximately 1.9 (range between valleys $=1.2-2.5$ ). This estimate compares well with the $R_{0}$ for canine zoonotic $V L$, which has been estimated to be $1.1,1.5,5.9$, and 11 in Spain, ${ }^{18}$ Italy, ${ }^{19}$ Brazil, ${ }^{14}$ and $\mathrm{Malta}^{20}$ respectively. Future analysis of human ACL data will have to determine to what extent canine transmission rates contribute to overall ACL transmission in Huánuco. $\mathrm{R}_{0}$ is probably the best indicator of the disease control problem. Because $R_{0} \approx 1.9$ is calculated from the mean incidence, the mean yearly effort (i.e., coverage or elimination) of a dog control intervention (e.g., vaccination, culling, or application of dog collars $)^{2,9,21}$ to ensure the elimination of $L$. (Viannia) spp. transmission (i.e., to reduce $\mathrm{R}_{0}<1$ ) can be estimated: $100\left(1-1 / R_{0}\right)=47 \%$. For example, in practical terms this would mean that $47 \%$ of the dogs would have to be collared in the first intervention year, assuming that insecticide-impregnated dog collars are $100 \%$ effective in protecting dogs from disease. ${ }^{21}$

One has to note that $\mathrm{R}_{0}$ does not measure the maximum seasonal potential for an ACL outbreak, nor can that quantity be obtained from seasonal or yearly incidence rates, i.e., one cannot use this data to develop a strategy to prevent a dog population from having epidemic outbreaks of L. (Viannia) infection. Moreover, the calculated $\mathrm{R}_{0}$ does not account for heterogeneous L. (Viannia) transmission by sand fly vectors, although entomologic and epidemiologic studies indicate that this is the case in Huánuco. ${ }^{12}$ When including heterogeneity in the distribution of sand fly vectors and hosts across endemic areas and using mathematical modeling, it has been shown that the $R_{0}$ may be in fact 2-4 times higher than the $R_{0}$ calculated on the assumption of homogeneous transmission. ${ }^{14,15}$ Modeling theory predicts that $20 \%$ of all hosts will account for $80 \%$ of the total transmission (i.e., the so-called $20 / 80$ rule), a hypothesis that has recently been confirmed by a field study showing that $20 \%$ of L. infantum-infected dogs account for more than $80 \%$ of the transmission to sand fly vectors. ${ }^{9}$ Clearly, this has implications for a dog control strategy, as in the case of Huánuco $\mathrm{R}_{0}$ could be as high as 7.6 and thus, the control effort to eliminate transmission in Huánuco could be as high as $87 \%$. The life expectancy of dogs in Huánuco was 3.0 years and the average time for a dog to acquire an infection was 3.4 years. As seen in Table 1, these figures vary between valleys, but generally appear to indicate that most dogs will not become infected during their lives. This in turn means that those dogs who become infected will account for most L. (Viannia) transmission to dogs (and humans). To account for heterogeneous transmission in the analyses, correlates of infectiousness will have to be known and a diagnostic test detecting highly infectious dogs will have to be available, neither of which is the case for either VL or ACL.

In summary, the results presented here, as well as the known association between dog ownership and risk of human $\mathrm{ACL}^{12}$ indicate that dogs, in the absence of alternative sylvatic reservoir hosts, are the ACL reservoir host in Huánuco, Peru. Epidemiologic analysis shows that a dog control strategy in Huánuco would be very feasible because the control effort to reduce $\mathrm{R}_{0}<1$ would be comparatively small. Such an intervention should also provide the ultimate proof in the incrimination of dogs as ACL reservoir hosts, as if such an intervention strategy were successful, it should have a significant epidemiologic impact on human ACL transmission.

Received July 1, 2003. Accepted for publication September 2, 2003.

Acknowledgments: We thank Wilder López Carrión, Luis Gomez, and Luis Leiva Lorenzo (Dirección Regional de Salud Huánuco, Peru) for their fantastic support in the field, as well as Alejandro Llanos-Cuentas and the Grupo de Estudios de Leishmaniasis (Universidad Peruana Cayetano Heredia, Peru) for logistical support. Rupert Quinnell (Leeds University, Leeds, United Kingdom) and Paul Coleman (London School of Hygiene and Tropical Medicine) made valuable comments on the manuscript.

Financial support: This study was funded by the Sir Halley Stewart Trust.

Authors' addresses: Richard Reithinger and Clive R. Davies, Disease Control and Vector Biology Unit, Department of Infectious and Tropical Diseases, London School of Hygiene and Tropical Medicine, Keppel Street, GB-London WC1E 7HT, United Kingdom, Telephone: 44-207-927-2350, Fax: 44-207-927-2164. E-mails: rreithinger@yahoo.co.uk and clive.davies@lshtm.ac.uk. Juan Canales Espinoza, Dirección Regional de Salúd Huánuco, Damaso Beraún 1016, Huánuco, Departamento de Huánuco, Peru, E-mail: jcanalesespinoza@hotmail.com.

\section{REFERENCES}

1. Davies CR, Reithinger R, Campbell-Lendrum D, Feliciangeli D, Borges R, Rodriguez N, 2000. The epidemiology and control of leishmaniasis in Andean countries. Cad Saude Publica. 16: 925-950. 
2. Ashford RW, 1996. Leishmaniasis reservoirs and their significance in control. Clin Dermatol 14: 523-532.

3. Reithinger R, Davies CR, 1999. Is the domestic dog (Canis familiaris) a reservoir host of American cutaneous leishmaniasis? A critical review of the current evidence. Am J Trop Med Hyg 61: 530-541.

4. Pirmez C, Coutinho SG, Marzochi MC, Nunes MP, Grimaldi G, 1988. Canine American cutaneous leishmaniasis: a clinical and immunological study in dogs naturally infected with Leishmania braziliensis braziliensis in an endemic area of Rio de Janeiro, Brazil. Am J Trop Med Hyg 38: 52-58.

5. Marco JD, Padilla AM, Diosque P, Fernandez MM, Malchiodi EL, Basombrio MA, 2001. Force of infection and evolution of lesions of canine tegumentary leishmaniasis in northwestern Argentina. Mem Inst Oswaldo Cruz 96: 649-652.

6. Moreno J, Alvar J, 2002. Canine leishmaniasis: epidemiological risk and experimental models. Trends Parasitol 18: 399-405.

7. Solano-Gallego L, Llull J, Ramos G, Riera C, Arboix M, Alberola J, Ferrer L, 2000. The Ibizian hound presents a predominantly cellular immune response against natural Leishmania infection. Vet Parasitol 90: 37-45.

8. Courtenay O, MacDonald DW, Lainson R, Shaw JJ, Dye C, 1994. Epidemiology of canine leishmaniasis: a comparative serological study of dogs and foxes in Amazon Brazil. Parasitology 109: 273-279.

9. Courtenay O, Quinnell RJ, Garcez LM, Shaw JJ, Dye C, 2002. Infectiousness in a cohort of Brazilian dogs: why culling fails to control visceral leishmaniasis in areas of high transmission. $J$ Infect Dis 186: 1314-1320.

10. Reithinger R, Lambson BE, Barker DC, Davies CR, 2000. Use of PCR to detect Leishmania (Viannia) spp. in dog blood and bone marrow. J Clin Microbiol 38: 748-751.

11. Reithinger R, Canales Espinoza J, Courtenay O, Davies CR, 2003. Evaluation of PCR as a diagnostic mass-screening tool to detect Leishmania (Viannia) spp. in domestic dogs (Canis familiaris). J Clin Microbiol 41: 1486-1493.

12. Reithinger R, Canales Espinoza J, Llanos-Cuentas A, Davies
CR, 2003. Domestic dog ownership: a risk factor for human infection with Leishmania (Viannia) spp. Trans $R$ Soc Trop Med Hyg 97: 141-145.

13. Williams B, Dye CM, 1994. Maximum-likelihood for parasitologists. Parasitol Today 10: 489-493.

14. Quinnell RJ, Courtenay O, Dye C, 1997. Epidemiology of canine leishmaniasis: transmission rates estimated from a cohort study in Amazon Brazil. Parasitology 115: 143-156.

15. Woolhouse ME, Dye C, Etard JF, Smith T, Charlwood JD, Garnett GP, Hagan P, Hii JL, Ndhlovu PD, Quinnell RJ, Watts CH, Chandiwana SK, Anderson RM, 1997. Heterogeneities in the transmission of infectious agents: implications for the design of control programs. Proc Natl Acad Sci USA 94: 338-342.

16. Davies CR, Llanos-Cuentas EA, Pyke SD, Dye C, 1995. Cutaneous leishmaniasis in the Peruvian Andes: an epidemiological study of infection and immunity. Epidemiol Infect 114: 297318.

17. Ramirez JL, Guevara P, 1997. Persistent infections by Leishmania (Viannia) braziliensis. Mem Inst Oswaldo Cruz 92: 333338.

18. Amela C, Mendez I, Torcal JM, Medina G, Pachon I, Canavate C, Alvar J, 1995. Epidemiology of canine leishmaniasis in the Madrid region, Spain. Eur J Epidemiol 11: 157-161.

19. Zaffaroni E, Rubaudo L, Lanfranchi P, Mignone W, 1999. Epidemiological patterns of canine leishmaniasis [correction of leishmaniosis] in Western Liguria (Italy). Vet Parasitol 81: 1119.

20. Dye C, Killick-Kendrick R, Vitutia MM, Walton R, KellickKendrick M, Harith AE, Guy MW, Canavate MC, Hasibeder G. 1992. Epidemiology of canine leishmaniasis: prevalence, incidence and basic reproduction number calculated from a cross-sectional survey on the island of Gozo, Malta. Parasitology 105: 35-41.

21. Reithinger R, Coleman PG, Alexander B, Vieira EP, Assis G, Davies CR, 2003. Are insecticide-impregnated dog collars a feasible alternative to culling as a strategy for controlling canine visceral leishmaniasis in Brazil? Int J Parasitol (in press). 